\title{
PERCEPÇÃO DA OBESIDADE JUVENIL ENTRE PROFESSORES DE EDUCAÇÃO FÍSICA NA EDUCAÇÃO BÁSICA
}

\author{
PERCEPTION OF YOUTH OBESITY AMONG PHYSICAL EDUCATION TEACHERS IN BASIC EDUCATION \\ PERCEPCIÓN DE LA OBESIDAD JUVENIL ENTRE LOS PROFESORES DE EDUCACIÓN FÍSICA EN LA \\ EDUCACIÓNBÁSICA
}

Jairo Antônio da Paixão'

(Educador Físico)

Caio Márcio Aguiar²

(Educador Físico)

Fabrício Sette Abrantes Silveira ${ }^{3}$

(Fisioterapeuta)

\begin{abstract}
1. Departamento de Educação Física da Universidade Federal de Viçosa (UFV), Viçosa, MG, Brasil.

2. Centro Desportivo da Universidade Federal de Ouro Preto (UFOP), Ouro Preto, MG, Brasil.

3. Departamento de Medicina e Enfermagem da Universidade Federal de Viçosa (UFV), Viçosa, MG, Brasil.
\end{abstract}

\section{Correspondência:}

Grupo de Estudos e Pesquisas Pedagógicas em Educação Física Departamento de Educação Física, Universidade Federal de Viçosa. Av. Peter Henry Rolfs, s/n, Campus Universitário. Viçosa, MG. Brasil. 36570-000

jairopaixao2004@yahoo.com.br

\begin{abstract}
RESUMO
Introdução: A obesidade pode ter início em qualquer idade, no entanto, tem ocorrido de modo crescente na população juvenil, o que, segundo casos registrados, é causa de inúmeros problemas de saúde. Objetivo: Analisar as percepções de professores de Educação Física atuantes em escolas de educação básica na cidade de Ouro Preto, MG, Brasil, com relação à obesidade juvenil e o papel da escola e da Educação Física como formas de controle. Métodos: Trata-se de estudo descritivo exploratório, no qual se utilizou a versão traduzida para o português do instrumento Perceptions of Youth Obesity and Physical Education Questionnaire em um grupo amostral de 15 professores licenciados em Educação Física de ambos os sexos (8 homens e 7 mulheres), atuantes em escolas de educação básica na cidade de Ouro Preto, MG, Brasil. Resultados: Os professores têm conhecimento das implicações da obesidade na saúde e qualidade de vida dos jovens, assim como da necessidade de intervenção. A partir da percepção desses professores, observa-se que a escola se configura como um espaço apropriado para abordar esse tema entre os jovens. Na perspectiva da educação para e pelo movimento, o professor de Educação Física pode auxiliar os jovens a promoverem mudanças significativas na forma física, a partir da adoção de um estilo de vida saudável e de orientações básicas de bons hábitos alimentares. Conclusão: A insuficiência da carga horária semanal de aulas de Educação Física para lidar com o tema da obesidade de forma sistemática, bem como suas implicações, é consenso entre esses professores.
\end{abstract}

Descritores: obesidade pediátrica; educação física e treinamento; docentes.

\section{ABSTRACT}

Introduction: Obesity can start at any age, however, has been increasing among the young population, which, according to reported cases, is the cause of numerous health problems. Objective: To analyze the perceptions of Physical Education teachers working in basic education schools in the city of Ouro Preto, Minas Gerais, Brazil, with respect to youth obesity and the role of schools and Physical Education as forms of control. Methods: This is a descriptive exploratory study, which used the instrument Perceptions of Youth Obesity and Physical Education Questionnaire in the version translated into Portuguese in a sample group of 15 licensed teachers in Physical Education of both sexes (8 men and 7 women) who work in basic education schools in the city of Ouro Preto, Minas Gerais, Brazil. Results: The teachers are aware of the implications of obesity on health and quality of life of young people, and the need for intervention. From the perception of these teachers, it is observed that the school is an appropriate place to address this issue among young people. From the perspective of education towards and by the movement, the Physical Education teacher can help young people to promote meaningful changes in physical form from the adoption of a healthy lifestyle and basic guidelines of good eating habits. Conclusion: The low weekly hour load of Physical Education classes to deal with the issue of obesity systematically and its implications is a consensus among these teachers.

Keywords: pediatric obesity; physical education and training; faculty.

\section{RESUMEN}

Introducción: La obesidad puede tener inicio a cualquier edad, sin embargo, ha ocurrido de modo creciente en la población juvenil, lo que, según los casos registrados, es causa de numerosos problemas de salud. Objetivo: Analizar las percepciones de los profesores de Educación Física que trabajan en escuelas de educación básica en la ciudad de Ouro Preto, Minas Gerais, Brasil, con respecto a la obesidad juvenil y el papel de las escuelas y la Educación Física como formas de control. Métodos: Se trata de un estudio exploratorio descriptivo, que utiliza la versión traducida al portugués del instrumento Perceptions of Youth Obesity and Physical Education Questionnaire en una muestra de 15 profesores licenciados en Educación Física de ambos sexos (8hombres y 7 mujeres), que trabajan en las escuelas de educación básica en la ciudad de Ouro Preto, Minas Gerais, Brasil. Resultados: Los profesores son conscientes de las consecuencias de la obesidad sobre la salud y la calidad de vida de los jóvenes y la necesidad de intervención. A partir de la percepción de estos profesores, se observa que la escuela es un espacio apropiado para abordar esta cuestión entre los jóvenes. Desde la perspectiva de la educación hacia y por el movimiento, el profesor de Educación Física puede ayudar a los jóvenes para promover cambios significativos en la forma física, a partir de la adopción de un estilo de vida saludabley directrices básicas de los 
buenos hábitos alimenticios. Conclusión: La carga horaria semanal baja de las clases de Educación Física para lidiar con el problema de la obesidad y sus consecuencias de manera sistemática es un consenso entre estos profesores.

Descriptores: obesidad pediátrica; educación y entrenamiento físico; docentes.

\section{INTRODUÇÃO}

Nos últimos anos, tamanha tem sido a prevalência da obesidade, tanto em populações de países desenvolvidos quanto em desenvolvimento, que muitos estudiosos a denominam como doença de proporção mundial, chegando, em alguns casos, entendê-la como uma pandemia global1-3. Trata-se de uma doença de origem multifatorial, que estabelece interação entre fatores genéticos, ambientais, socioeconômicos, endócrinos e metabólicos ${ }^{2}$. Dentre os fatores ambientais, destacam-se aqueles relacionados ao aumento do consumo de açúcares, alimentos industrializados e ingestão insuficiente de frutas e hortaliças, além de redução progressiva da prática de atividades físicas, combinada com maior tempo em atividades de baixa intensidade, como, por exemplo, assistir televisão e usar o computador.

Pesquisas têm apontado para a prevalência do sobrepeso e da obesidade entre crianças e adolescentes ${ }^{5-10}$. Chama a atenção o fato de que, nas referidas fases da vida, menos de $10 \%$ dos casos de sobrepeso e obesidade decorrem de fatores endócrinos ${ }^{2,11,12}$ e ainda, estudos longitudinais mostram que a obesidade da infância tende a prevalecer na fase adulta da vida13,14.

Numa perspectiva que sinaliza estratégias educativas com vistas a minimizar os efeitos negativos provenientes de estilos de vida que aviltam a saúde e a qualidade de vida da população juvenil, dentre os espaços e instituições sociais, a escola configura-se como lócus privilegiado para abordar essa problemática ${ }^{15}$.

Mais especificamente, a Educação Física, área do conhecimento que apresenta o movimento humano como eixo norteador das diversas práticas corporais, possui o potencial no trabalho de conscientização dos alunos sobre a importância da adoção de um estilo de vida ativo. Trata-se de uma valiosa ação educativa na qual se abre espaço para abordar, por exemplo, os malefícios da obesidade, como uma situação que pode se apresentar como contraponto na aquisição e manutenção da saúde e qualidade de vida ${ }^{16}$.

Neste panorama, o objetivo desse estudo foi analisar a percepção dos professores de Educação Física em relação à obesidade juvenil e o papel da escola e da disciplina Educação Física no controle dessa problemática.

\section{MATERIAIS E MÉTODOS}

O presente trabalho adotou os procedimentos metodológicos de um estudo descritivo-exploratório ${ }^{17,18}$. O grupo amostral foi constituído de 15 professores licenciados em Educação Física de ambos os sexos (8 homens e 7 mulheres), atuantes em escolas de educação básica na cidade de Ouro Preto, MG, Brasil. Buscando assegurar uma amostra representativa, o número de participantes foi arbitrado pelo prazo de três meses, definido para a realização da fase de coleta de dados no projeto da presente investigação.

Como critérios de inclusão dos sujeitos no grupo amostral, foram considerados professores licenciados em Educação Física atuantes no ensino fundamental II e ensino médio em escolas de educação básica (rede pública e privada de ensino), e, após tomarem ciência dos objetivos e natureza da pesquisa, a assinatura do Termo de Consentimento e Livre Esclarecido (TCLE). Consideraram-se, como critérios de exclusão, os sujeitos que não atenderam às condições descritas.

Para a coleta de dados, foi utilizada a versão traduzida para o português do instrumento Perceptions of Youth Obesity and Physical Education Questionnaire $^{19}$, para avaliar a percepção do papel da Educação Física na diminuição do sobrepeso e obesidade juvenil. Esse questionário contém 12 itens que se referem a fatores que podem contribuir para a obesidade e outros 30 itens sobre o problema da obesidade juvenil e o papel da escola e da Educação Física em lidar com os problemas da obesidade. Na operacionalização das variáveis contidas nos itens, o questionário estrutura-se a partir da escala Likert de cinco pontos, em que o informante poderia marcar uma alternativa ( $1=$ discordo plenamente; 2 = discordo; 3 = indiferente; 4 = concordo; 5 = concordo plenamente) em ordem de importância, de acordo com a percepção dos temas relacionados à obesidade na fase juvenil. Para fins de apresentação dos resultados, as categorias 1 e 2 foram consideradas negativas, a 3 neutra e as categorias 4 e 5 positivas.

A tradução para o português do instrumento utilizado para a coleta de dados foi realizada pelo pesquisador, que buscou assegurar a veracidade da versão original, visando, sobretudo, evitar e/ou minimizar risco na forma de interpretação por parte dos sujeitos participantes do estudo.

Como parte dos procedimentos adotados para a coleta de dados foram realizados contatos prévios com os sujeitos e agendamento dos encontros para a aplicação dos questionários. A coleta de dados ocorreu no período de novembro de 2014 a fevereiro de 2015, nas escolas onde os professores participantes atuavam.

Nos procedimentos adotados para o tratamento dos dados coletados, tendo em vista os itens que constituíam o questionário Perceptions of Youth Obesity and Physical Education Questionnaire ${ }^{19}$ foram elaboradas três categorias denominadas: percepção da obesidade juvenil (Tabela 1) papel da escola (Tabela 2) e papel da Educação Física (Tabela 3), nas quais esses itens foram agrupados de acordo com o grau de afinidade com a categoria correspondente.

O termo juvenil, empregado neste artigo, se refere aos sujeitos de ambos os sexos que estão na faixa etária denominada adolescência que, segundo a Organização Mundial da Saúde, ${ }^{5}$ se encontram entre o limite cronológico de 10 e 19 anos de idade. Para efeito do objeto de investigação a que se propôs a presente de pesquisa, foi considerado juvenil, adolescentes que se encontram na faixa etária de 11 a 17 anos (ensino fundamental Il e ensino médio).

O estudo foi aprovado pelo Comitê de Ética em Pesquisa com seres humanos da Universidade Federal de Ouro Preto, MG, Brasil, parecer número 291.921 de 29/05/2013.

\section{RESULTADOS}

Os resultados encontram-se organizados em três categorias de análise estabelecidas a partir dos objetivos do estudo: a primeira busca analisar a percepção dos professores com relação à obesidade juvenil; a segunda aborda o papel da escola sobre a obesidade na percepção dos professores e a terceira, a percepção dos professores sobre o papel da Educação Física no controle da obesidade.

\section{A percepção dos professores com relação à obesidade juvenil}

Os resultados, apresentados na Tabela 1, versam sobre a percepção dos professores licenciados em Educação Física sobre a obesidade, tendo como eixos norteadores a relevância da problemática, sua correlação com a saúde e prevalência de possíveis resultados obtidos por meio de programas voltados ao emagrecimento.

Os resultados mostram um consenso entre os professores entrevistados (93\%) sobre a relevância do tema acerca do aumento acentuado da obesidade entre a população juvenil nos últimos tempos. Nas tentativas de 
perder peso por meio de programas destinados a essa finalidade, acredita-se que o insucesso, numa primeira tentativa implica, negativamente nas tentativas posteriores. Ao serem questionados sobre a prevalência dos resultados obtidos em programas voltados à perda de peso, quase a totalidade dos participantes (mais de 96,5\%) reconhece que os jovens que contam com apoio e ajuda apropriada serão capazes, pois não somente irão obter perdas mais significativas de peso, bem como de manter essas perdas ao longo da vida. Não obstante a isso, os participantes relacionaram o peso ideal na fase juvenil como uma importante condição para a saúde.

\section{O papel da escola sobre a obesidade}

A Tabela 2 apresenta dados relacionados à percepção dos professores sobre o papel da escola no controle da obesidade juvenil. Foram

Tabela 1. Percepção sobre a obesidade juvenil

\begin{tabular}{|c|c|c|c|c|c|}
\hline \multirow{2}{*}{ Variáveis consideradas } & \multicolumn{5}{|c|}{ Escala de LIKERT (\%) } \\
\hline & 1 & 2 & 3 & 4 & 5 \\
\hline $\begin{array}{l}\text { A obesidade juvenil está se convertendo em um tema } \\
\text { cada vez mais relevante. }\end{array}$ & 7 & -- & -- & 53 & 40 \\
\hline $\begin{array}{l}\text { O fracasso na hora de perder peso, após participar de } \\
\text { um programa destinado a esse fim, reforça as crenças } \\
\text { do jovem de que existe uma pequena oportunidade de } \\
\text { conseguir essa perda. }\end{array}$ & 22 & 7 & 28 & 43 & -- \\
\hline $\begin{array}{c}\text { Ter um peso normal é muito importante para a saúde dos } \\
\text { jovens. }\end{array}$ & -- & -- & -- & 47 & 53 \\
\hline $\begin{array}{l}\text { As pessoas obesas são sexualmente menos atraentes que } \\
\text { as pessoas magras. }\end{array}$ & 20 & 40 & 20 & 13 & 7 \\
\hline $\begin{array}{c}\text { A obesidade juvenil é uma causa significativa de rejeição } \\
\text { entre iguais. }\end{array}$ & 13 & 13 & 7 & 60 & 7 \\
\hline Muitos jovens superarão sua obesidade. & -- & 20 & 40 & 40 & -- \\
\hline Nunca sairia com uma pessoa obesa. & 71 & 21 & $\begin{array}{ll}- \\
-\end{array}$ & 7 & -- \\
\hline $\begin{array}{l}\text { Com a ajuda apropriada, muitos jovens obesos são } \\
\text { capazes de perder uma considerável quantidade de peso. }\end{array}$ & -- & -- & -- & 60 & 40 \\
\hline $\begin{array}{c}\text { No geral, as pessoas obesas são mais preguiçosas que as } \\
\text { pessoas magras. }\end{array}$ & 26 & 20 & 7 & 33 & 13 \\
\hline $\begin{array}{l}\text { Com a ajuda apropriada, muitos jovens obesos que } \\
\text { perderam peso são capazes de manter essa perda. }\end{array}$ & -- & 7 & -- & 67 & 26 \\
\hline
\end{tabular}

1=Discordo plenamente; 2=Discordo; 3=Indiferente; 4=Concordo; 5=Concordo plenamente

Tabela 2. Papel da escola.

\begin{tabular}{|c|c|c|c|c|c|}
\hline \multirow{2}{*}{ Variáveis consideradas } & \multicolumn{5}{|c|}{ Escala de LIKERT (\%) } \\
\hline & 1 & 2 & 3 & 4 & 5 \\
\hline $\begin{array}{c}\text { As escolas deveriam dispor de cardápios com } \\
\text { baixas calorias. }\end{array}$ & -- & 7 & -- & 53 & 40 \\
\hline $\begin{array}{l}\text { Os pais não deveriam converter a escola em um } \\
\text { lugar para o tratamento da obesidade juvenil. }\end{array}$ & 20 & -- & -- & 60 & 20 \\
\hline $\begin{array}{c}\text { A bulimia e a anorexia nervosa são problemas } \\
\text { mais sérios dentro do contexto escolar que a } \\
\text { obesidade. }\end{array}$ & 14 & 43 & 21 & 14 & 7 \\
\hline $\begin{array}{l}\text { As escolas não se envolvem o suficiente para } \\
\text { ajudar a combater o problema da obesidade. }\end{array}$ & 7 & 7 & 7 & 53 & 26 \\
\hline $\begin{array}{c}\text { Todas as escolas deveriam dispor de aulas de } \\
\text { E F especificamente pensadas para os jovens } \\
\text { obesos. }\end{array}$ & 13 & 47 & 7 & 26 & 7 \\
\hline $\begin{array}{c}\text { As escolas deveriam dispor de programas de } \\
\text { controle de peso destinados a tratar a obesida- } \\
\text { de juvenil. }\end{array}$ & -- & 14 & 7 & 57 & 21 \\
\hline $\begin{array}{l}\text { Deveriam eliminar-se das cantinas das escolas } \\
\text { os alimentos ricos em gordura, balas e doces. }\end{array}$ & 7 & 13 & 7 & 53 & 20 \\
\hline $\begin{array}{c}\text { O professor regente deveria fazer, na escola, } \\
\text { o controle e tratamento da obesidade nas } \\
\text { crianças. }\end{array}$ & 33 & 40 & 7 & 13 & 7 \\
\hline $\begin{array}{c}\text { As escolas deveriam dispor de matérias curricu- } \\
\text { lares que contenham conteúdos sobre nutrição } \\
\text { e controle de peso. }\end{array}$ & -- & -- & 7 & 53 & 40 \\
\hline
\end{tabular}

consideradas as variáveis: o tratamento da problemática nas instituições e medidas a serem tomadas para a melhoria no quadro atual.

Os resultados demonstram que, no tocante aos alimentos distribuídos nas escolas, a maioria dos sujeitos (93\%) acredita que os cardápios deveriam conter apenas alimentos de baixas calorias. Por outro lado, com relação à postura a ser adotada pela escola no sentido de amenizar o quadro atual de obesidade juvenil, os participantes (80\%) não concordam com que as famílias convertam a escola em um espaço para se tratar a obesidade juvenil. Por outro lado, mais de $79 \%$ desses mesmos sujeitos afirmam que as escolas não têm se envolvido o suficiente com a questão da obesidade entre seus alunos. Ainda com relação ao papel da escola mediante a obesidade juvenil, 73\% dos participantes não concordam com que seja delegada ao professor regente a responsabilidade nas ações visando ao tratamento da obesidade entre os seus alunos na sala de aula. Diante da percepção dos entrevistados sobre o papel da escola no controle da obesidade juvenil, destaca-se a ideia de que a escola deveria oferecer conteúdos curriculares relacionados à nutrição e ao controle do peso corporal.

\section{O papel da Educação Física no controle da obesidade}

Destacando o objetivo final desta pesquisa, a Tabela 3 apresenta a forma como os sujeitos percebem a Educação Física no controle à obesidade e no fomento de um estilo de vida saudável dos alunos na fase juvenil. Nesse sentido, foram destacadas as seguintes variáveis: duração das aulas, função do professor da disciplina com relação ao problema, programação de atividades na escola e formação no curso de licenciatura em Educação Física para lidar com as questões relacionadas à obesidade juvenil.

A carga horária semanal das aulas de Educação Física - geralmente duas aulas de 50 minutos - é percebida pela maioria dos professores entrevistados (72\%) como insuficiente para que ocorram alterações corporais significativas nas crianças e jovens. Apesar disso, mais da metade dos participantes (66\%) discorda de que o professor da disciplina Educação Física deve fazer o controle da obesidade na escola e servir de modelo para os alunos, mantendo o seu peso dentro dos padrões considerados normais. No entanto, ao se postular sobre a possibilidade de direcionar as aulas de Educação Física no sentido de enfatizar o cuidado com a forma física dos jovens ao longo da vida, as percepções se dividem em duas posições:

Tabela 3. Papel da educação física

\begin{tabular}{|c|c|c|c|c|c|}
\hline \multirow{2}{*}{ Variáveis consideradas } & \multicolumn{5}{|c|}{ Escala de LIKERT (\%) } \\
\hline & 1 & 2 & 3 & 4 & 5 \\
\hline $\begin{array}{l}\text { Não se dispõe do tempo suficiente nas aulas de E F para } \\
\text { ajudar os jovens a melhorarem sua forma física. }\end{array}$ & 13 & 7 & 7 & 46 & 26 \\
\hline $\begin{array}{c}\text { Aconselhar os jovens e seus pais sobre os programas } \\
\text { de exercícios para perder peso é uma atividade muito } \\
\text { gratificante. }\end{array}$ & -- & -- & 14 & 50 & 36 \\
\hline $\begin{array}{c}\text { A formação recebida não me preparou de forma adequada } \\
\text { para elaborar um programa de atividades para ajudar os } \\
\text { jovens a reduzir seu peso. }\end{array}$ & 26 & 26 & 13 & 33 & -- \\
\hline $\begin{array}{l}\text { O professor de EF deveria fazer, na escola, o controle e } \\
\text { tratamento da obesidade nos juvenis. }\end{array}$ & 33 & 33 & 7 & 20 & 7 \\
\hline $\begin{array}{l}\text { Acredito que sou competente na hora de prescrever um } \\
\text { programa de exercícios destinado à perda de peso para } \\
\text { alunos obesos. }\end{array}$ & -- & 13 & 7 & 60 & 20 \\
\hline $\begin{array}{c}\text { Conceber programas de atividades para perder peso e } \\
\text { aconselhar tanto os jovens quanto seus pais sobre tais } \\
\text { aspectos é uma tarefa muito difícil. }\end{array}$ & 7 & 40 & 13 & 27 & 13 \\
\hline $\begin{array}{l}\text { Acredito que os professores de EF deveriam servir de } \\
\text { modelo, sendo exemplo e mantendo um peso normal. }\end{array}$ & 7 & 40 & 13 & 20 & 20 \\
\hline $\begin{array}{l}\text { Os alunos deveriam receber aulas de EF centradas no } \\
\text { cuidado com a forma física ao longo de todo o ciclo de vida. }\end{array}$ & 13 & 20 & 26 & 26 & 13 \\
\hline $\begin{array}{c}\text { Muitas das aulas de EF não são elaboradas para } \\
\text { proporcionar hábitos de vida e padrões de exercício que } \\
\text { ajudem a controlar o peso. }\end{array}$ & 7 & 7 & 7 & 53 & 26 \\
\hline
\end{tabular}


39\% concordam e 33\% discordam com tal atribuição à Educação Física. Faz-se necessário levar em consideração um terceiro grupo, composto por $28 \%$, que optou por não se manifestar quanto à referida questão. No que se refere à formação inicial no curso de licenciatura, 52\% afirmaram que o curso tinha lhes preparado para elaborar programas de atividades para reduzir o excesso de peso em jovens, enquanto 33\% negaram ter recebido essa preparação no curso de formação. Trata-se de um achado que chama a atenção haja vista a principal finalidade do curso de licenciatura que, por um lado, prepara o sujeito para atuar na educação básica, por outro dista de procedimentos relacionados à elaboração de programas voltados para perda de peso corporal, comumente abordado no curso de bacharelado em Educação Física.

\section{DISCUSSÃO}

Os resultados obtidos na presente investigação, em concordância com outros estudos ${ }^{6,11,16,20,21}$ revelaram que os sujeitos entrevistados percebem a relevância do tema obesidade juvenil nos dias atuais, e ressaltam a importância da adequação da composição corporal ao percentil para a faixa etária no sentido de se evitar doenças relacionadas.

A pesquisa mostrou que a obesidade causa rejeição entre iguais, podendo acarretar atitudes preconceituosas desde a fase infantil. Essa mesma situação é mostrada em estudos sobre o tema ${ }^{11,16}$. Esse tipo de rejeição pode estar relacionado à forma de perceber a pessoa obesa como menos atraente, menos vaidosa e mais preguiçosa em comparação a pessoa que se encontra com o peso corporal adequado com sua faixa etária e altura. Essa forma preconceituosa de perceber o outro resulta de um padrão de beleza corporal que prevalece na sociedade ${ }^{11}$.

De maneira geral, a percepção dos professores de Educação Física sobre a pessoa obesa não revelou atitudes negativas. Os dados se mostraram favoráveis no que se refere à atuação desses professores em programas destinados à prevenção e ao controle da obesidade juvenil na escola.

No que se refere à superação da obesidade entre os jovens, os participantes da pesquisa acreditam que, com ajuda apropriada, os jovens superarão sua obesidade e serão capazes de manter um peso ideal para a faixa etária. Isso porque, estudos relacionados ao tema mostram que a perda de peso corporal torna-se possível mediante mudanças no estilo de vida, alimentação e exercícios físicos orientados, atuando na regulação do balanço energético ${ }^{20,22}$. No entanto, vale ressaltar que os professores entrevistados não concordam que as aulas de Educação Física tenham como principal finalidade controlar índices excessivos de adiposidade corporal entre os alunos. Esse mesmo posicionamento se aplica à escola. Esse resultado coaduna com estudos que comprovaram que ainda que a escola possa ser considerada um espaço para instalação e efetivação de programas e políticas públicas para o tratamento da obesidade juvenil, os professores de Educação Física opõem-se a ideia da sua conversão exclusiva para tal finalidade ${ }^{20}$. Na perspectiva que sinaliza estratégias educativas para a minimização dos efeitos negativos provenientes da obesidade, é consenso entre os professores a necessidade de eliminar das cantinas alimentos ricos em gordura, balas e doces. Tal crença encontra-se em consonância com o Programa Nacional de Alimentar Escolar - PNAE (FNDE, 2013)23, que define o oferecimento, pela escola, em torno de 20\% da ingestão necessária tanto de calorias quanto de nutrientes aos alunos. Esse posicionamento corrobora com estudos realizados por Kac et al..$^{24}$ que apontam uma mudança de padrão alimentar nos últimos anos.

Ao questionar os sujeitos se as escolas deveriam dispor das aulas de Educação Física para atendimento dos jovens obesos, a maioria discordou dessa possibilidade. Esses resultados podem estar relacionados ao fato da percepção desses professores sobre os demais objetivos e finalidades atribuídos à Educação Física como prática de intervenção na escola de educação básica. Esses objetivos incluem para além dos conhecimentos sobre o corpo e suas práticas, àquelas relacionadas às diferentes manifestações culturais produzidas historicamente pelo homem, dentre outras que também se fazem importantes a ser tratadas nas aulas de Educação Física escolar.

\section{CONCLUSÃO}

Diante das constatações obtidas nesta investigação e considerando as suas limitações metodológicas, é possível afirmar que os professores licenciados em Educação Física atuantes na educação básica, reconhecem a necessidade de intervenção para prevenir e controlar a obesidade entre jovens. Constatou-se também a importância das instituições formadoras nesse processo, com o implemento de projetos e programas a fim de contribuir para minimizar o crescente quadro de sobrepeso e obesidade no Brasil.

Tendo em vista os resultados apresentados nesta pesquisa, sugerem-se novas investigações sobre o tema, incluindo observação de aulas, análise aprofundada de planejamento e de matrizes curriculares dos cursos de formação e, ainda, possibilidades de acesso e articulação dos saberes relacionados à obesidade e seus procedimentos por professores licenciados em Educação Física na escola.

\section{AGRADECIMENTOS}

Os autores agradecem o apoio concedido pelo Conselho Nacional de Pesquisa (CNPq) na condução da presente pesquisa.

Todos os autores declararam não haver qualquer potencial conflito de interesses referente a este artigo.

CONTRIBUIÇÕES DOS AUTORES: Cada autor contribuiu individual e significativamente para o desenvolvimento do manuscrito. JAP (0000-0003-1413-9081)* contribuiu na redação, normas da revista, tratamento, análise e discussão dos dados. CMA (0000-0001-6529-2022)* realizou o levantamento bibliográfico em bancos de dados, coleta de dados e redação. FSAS (0000-0002-4434-6519)* realizou a análise quantitativa dos dados e revisão do manuscrito. *ORCID (Open Researcher and Contributor ID).

\section{REFERÊNCIAS}

1. Bertoletti J, Garcia-Santos, SC. Avaliação do estresse na obesidade infantil. Psico. 2012;43(1):32-38

2. Marchi-Alves LM, Yagui CM, Rodrigues CS, Mazzo A, Rangel EML, Girão FB. Obesidade infantil ontem e hoje: importância da avaliação antropométrica pelo enfermeiro. Esc Anna Nery. 2011;15(2):238-44

3. Pereira A1, Guedes AD, Verreschi IT, Santos RD, MartinezTL. A Obesidade e sua Associação com os Demais Fatores de Risco Cardiovascular em Escolares de Itapetininga, Brasil. Arq Bras Cardiol. 2009;93(3):253-60.

4. Enes CC, Slater B. Obesidade na adolescência e seus principais fatores determinantes. Rev Bras Epidemiol. 2010;13(1):163-71.

5. World Health Organization (WHO). World Health Statistics 2014: Part II Highlighted topics. Geneva: Who Library Cataloguing-in-Publication Data. [cited 2015 may 23]. Available from: http://www.who.int.

6. Garcia EA, Carías D, Valery MP, Naddaf G, DomínguezZ. Exceso de peso, resistencia a la insulina y dislipidemia en adolescentes. Acta Bioquími Clín Latinoam. 2012. [acesso em 2015 mar 15];46(3):365-73. Disponível em: http://www.scielo.org.ar/scielo.php?pid=03252957\&script=sci_serial
7. Mascarenhas LPG, Modesto MJ, Amer NM, Boguszewski MCS, Lacerda Filho L, Prati FS. Influência do excesso de peso dos pais em relação ao sobrepeso e obesidade dos filhos. Pensar a Prática. Goiânia. 2013;16(2): 519-32.

8. Conde WL, Borges C. O risco de incidência e persistência da obesidade entre adultos Brasileiros segundo seu estado nutricional ao final da adolescência. Rev Bras de Epidemiol. 2011;14(Supll 1):71-9.

9. Mello ED, LuftVC, Meyer F. Obesidade infantil: como podemos ser eficazes? J Pediatr (Rio J). 2004;80(3):173-82

10. Gentile DA, Welk G, Eisenmann JC, Reimer RA, Walsh DA, Russell DW. et al. Evaluation of a multiple ecological level child obesity prevention program: Switch what you Do, View, and Chew. BMC Med. 2009;7(1):49.

11. Costa MAP, Souza MA, Oliveira VM. Obesidade infantil e bullying: a ótica dos professores. Educ Pesqui. 2012;38(3):653-65.

12. Nascimento VG, Silva JP, Bertoli CJ, Abreu LC, Valenti VE, Leone C. Prevalence of overweight preschoo children in public day care centers: a cross-sectional study. São Paulo Med J. 2012;130(4):225-9.

13. Must A. Morbidity and mortality associated with elevated body weight in children and adolescents. 
Am J Clin Nutr. 1996 [cited 2015 apr 15];63(Suppl 3):S444-7. Available from: http://ajcn.nutrition.org/ content/63/3/445S.full.pdf + html.

14. Rössner S. Childhood obesity and adulthood consequenses. Acta Paediatr. 1998 [cited 2015 apr 16];87(1):1-5. Available from: http://www.ncbi.nlm.nih.gov/pubmed/9510438.

15. Procter KL, Rudolf MC, Feltbower RG, Levine R, Connor A, Robinson M, et al. Measuring the school impact on child obesity. Soc Sci Med. 2008;67(2):341-9.

16. Greenleaf C, Weiller K. Perceptions of youth obesity among physical educators. Soc Psychol Educ. 2005;8(4)407-23.

17. Malhotra NK. Pesquisa de marketing: uma orientação aplicada. 6a ed. Porto Alegre: Bookman, 2011.

18. Thomas JR, Nelson JK, Silverman SJ. Métodos de pesquisa em atividade física. $6^{a}$ ed. Porto Alegre: Artmed, 2012.

19. Price JH, Desmond SM, Ruppert ES. Elementary physical education teachers' perceptions of childhood obesity. Health Education. 1990;21(8) 26-32

20. Galatti LR, Seoane MA, Paes RR. Pedagogia do Esporte e Obesidade: perspectivas para um estilo de vida saudável a partir da adequada iniciação esportiva na infância. Pensar Prát. 2012;15(2)452-67.

21. Azevedo FR, Ikeoka D, Caramelli B. Effects of intermittent fasting on metabolism in men. Rev Assoc Med Bras. 2013;59(2):167-73.

22. Fernandes MM, Penha DSG, Braga FA. Obesidade infantil em crianças da rede pública de ensino: prevalência e consequências para flexibilidade, força explosiva e velocidade. Rev Educ Fís/UEM. 2012;23(4):629-34.

23. Programa Nacional de Alimentação Escolar (PNAE). Fundo Nacional de desenvolvimento da Educação. Ministério da Educação. Resolução/CD/FNDE no 26, 17 de junho de 2013. (acesso em 2015 mai 18). Disponível em: http://www.fnde.gov.br/fnde/legislacao/resolucoes/ item/4620-resolu\%C3\%A7\%C3\%A3o-cd-fnde-n\%C2\%BA-26,-de-17-de-junho-de-2013.

24. Kac G, Sichieri R, Gigante DP. Epidemiologia nutricional. Rio de Janeiro: Editora Fiocruz/Ateneu, 2007. 